\title{
A Duality Result for Moduli Spaces of Semistable Sheaves Supported on Projective Curves
}

\author{
MARio MAicAN (*)
}

ABSTRACT - We show that the map sending a sheaf to its dual gives an isomorphism of the moduli space of semistable sheaves with fixed multiplicity and Euler characteristic and supported on projective curves to the moduli space of semistable sheaves of dimension one on the projective space with the same multiplicity but with opposite Euler characteristic.

Let $k$ be an algebraically closed field of characteristic zero. Let $\mathbb{P}^{n}$ be the projective space of dimension $n$ over $k$. We recall that a coherent algebraic sheaf $\mathcal{F}$ on $\mathbb{P}^{n}$ has support of dimension one if and only if its Hilbert polynomial $P_{\mathcal{F}}$ has degree one that is, if we can write $P_{\mathcal{F}}(m)=r m+\chi$. Here $r=r(\mathcal{F})$ is a positive integer called the multiplicity of $\mathcal{F}$, while $\chi$ is the Euler characteristic $\chi(\mathcal{F})=h^{0}(\mathcal{F})-h^{1}(\mathcal{F})$.

We fix integers $r \geq 1$ and $\chi$ and we denote by $\mathrm{M}_{\mathrm{P}^{n}}(r, \chi)$ the moduli space of semistable sheaves on $\mathbb{P}^{n}$ with Hilbert polynomial $P(m)=r m+\chi$. In this paper we will prove that $\mathrm{M}_{\mathbb{P}^{n}}(r, \chi)$ and $\mathrm{M}_{\mathrm{P}^{n}}(r,-\chi)$ are isomorphic. The isomorphism maps a point represented by $\mathcal{F}$ to the point represented by the dual sheaf $\mathcal{E} x t^{n-1}\left(\mathcal{F}, \omega_{\mathbb{P}^{n}}\right)$. We will use the concept of semistability due to Gieseker and defined in terms of the lexicographic order on the coefficients of the reduced Hilbert polynomial, cf. definition 1.2.4 in [12]. We recall that a sheaf $\mathcal{F}$ on $\mathbb{P}^{n}$ with one-dimensional support is semistable (stable) if and only if it is pure, meaning that there are no subsheaves with support of dimension zero, and for every proper subsheaf $\mathcal{E} \subset \mathcal{F}$ we have

$$
\frac{\chi(\mathcal{E})}{r(\mathcal{E})} \leq(<) \frac{\chi(\mathcal{F})}{r(\mathcal{F})}
$$

It was first noticed in [5] that $\mathcal{F} \longrightarrow \mathcal{E} x t^{1}\left(\mathcal{F}, \omega_{\mathrm{p}^{2}}\right)$ gives a birational map from $\mathrm{M}_{\mathrm{p}^{2}}(r, \chi)$ to $\mathrm{M}_{\mathrm{p}^{2}}(r,-\chi)$ when $r$ and $\chi$ are mutually prime.

(*) Indirizzo dell'A.: Str. Avrig 9-19, Ap. 26, Bucharest, Romania.

E-mail: m-maican@wiu.edu 
We begin by recalling the Beilinson free monad. Let $\mathcal{F}$ be a sheaf on $\mathbb{P}^{n}$. A monad for $\mathcal{F}$ is a sequence of sheaves

$$
0 \longrightarrow \mathcal{C}^{-p} \longrightarrow \ldots \longrightarrow \mathcal{C}^{0} \longrightarrow \ldots \longrightarrow \mathcal{C}^{q} \longrightarrow 0
$$

which is exact, except at $\mathcal{C}^{0}$, where the cohomology is $\mathcal{F}$. If each $\mathcal{C}^{i}$ is a direct sum of line bundles we talk of a free monad. In the sequel $\mathcal{F}$ will be a coherent sheaf on $\mathbb{P}^{n}$. According to [1], cf. also [4], there is a free monad for $\mathcal{F}$

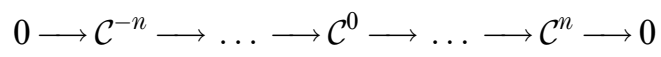

with

$$
\mathcal{C}^{i}=\bigoplus_{0 \leq p \leq n} \mathrm{H}^{i+p}\left(\mathcal{F} \otimes \Omega^{p}(p)\right) \otimes \mathcal{O}(-p) .
$$

Lemma 1. Consider a free monad for $\mathcal{F}$

$$
0 \longrightarrow \mathcal{A}^{-n} \longrightarrow \ldots \longrightarrow \mathcal{A}^{0} \longrightarrow \ldots \longrightarrow \mathcal{A}^{n} \longrightarrow 0
$$

with $\mathcal{A}^{i}=\underset{0 \leq p \leq n}{\bigoplus} W_{i p} \otimes \mathcal{O}(-p)$, where $W_{i p}$ are vector spaces over $k$ of $d i$ mension $w_{i p}$. Then $w_{i p}=\mathrm{h}^{i+p}\left(\mathcal{F} \otimes \Omega^{p}(p)\right)$ for all $i$ and $p$ if and only if each map $W_{i, p} \otimes \mathcal{O}(-p) \longrightarrow W_{i+1, p} \otimes \mathcal{O}(-p)$ occuring in the monad is zero.

Proof. The two spectral sequences 'E and "E converging to the hypercohomology of $\mathcal{A}^{*} \otimes \Omega^{j}(j)$ are given by

$$
\begin{aligned}
{ }^{\prime} \mathrm{E}_{2}^{p q} & =\mathrm{H}^{p}\left(\mathbb{P}^{n}, \mathcal{H}^{q}\left(\mathcal{A}^{*} \otimes \Omega^{j}(j)\right)\right), \\
{ }^{\prime} \mathrm{E}_{1}^{p q} & =\mathrm{H}^{q}\left(\mathbb{P}^{n}, \mathcal{A}^{p} \otimes \Omega^{j}(j)\right) .
\end{aligned}
$$

By hypothesis $\mathcal{H}^{q}\left(\mathcal{A}^{*} \otimes \Omega^{j}(j)\right)$ is $\mathcal{F} \otimes \Omega^{j}(j)$ for $q=0$ and is zero for $q \neq 0$. Thus

$$
{ }^{\prime} \mathrm{E}_{2}^{p q}= \begin{cases}\mathrm{H}^{p}\left(\mathcal{F} \otimes \Omega^{j}(j)\right), & \text { for } q=0, \\ 0 & \text { for } q \neq 0,\end{cases}
$$

which shows that 'E degenerates at level two. The differentials in the second spectral sequence ${ }^{\prime \prime} \mathrm{E}_{1}^{p, q} \longrightarrow{ }^{\prime \prime} \mathrm{E}_{1}^{p+1, q}$ are induced on cohomology by the maps $\mathcal{A}^{p} \otimes \Omega^{j}(j) \longrightarrow \mathcal{A}^{p+1} \otimes \Omega^{j}(j)$. According to Bott's formulas on p. 8 in [15] we have $\mathrm{h}^{q}\left(\Omega^{j}(j-i)\right)=0$ for $q \neq j, 0 \leq i \leq n$ or for $q=j, i \neq j$, $0 \leq i \leq n$. Thus " $\mathrm{E}_{1}^{p q}=0$ if $q \neq j$ and we have the identification

$$
{ }^{\prime \prime} \mathrm{E}_{1}^{p j}=W_{p j} \otimes \mathrm{H}^{j}\left(\mathbb{P}^{n}, \Omega^{j}\right) \simeq W_{p j} .
$$

The differentials $f_{p j}:$ : $\mathrm{E}_{1}^{p, j} \longrightarrow " \mathrm{E}_{1}^{p+1, j}$ are identified with the maps from the $\operatorname{monad} W_{p, j} \otimes \mathcal{O}(-j) \longrightarrow W_{p+1, j} \otimes \mathcal{O}(-j)$. 
Assume now that all maps $f_{p j}$ are zero. Then "E degenerates at its first term and, comparing ' $\mathrm{E}$ with " $\mathrm{E}$, we get

$\mathrm{h}^{p}\left(\mathcal{F} \otimes \Omega^{j}(j)\right)=\operatorname{dim}\left({ }^{\prime} \mathrm{E}_{2}^{p 0}\right)=\operatorname{dim}\left({ }^{\prime} \mathrm{E}_{\infty}^{p}\right)=\operatorname{dim}\left({ }^{\prime \prime} \mathrm{E}_{\infty}^{p}\right)=\operatorname{dim}\left({ }^{\prime \prime} \mathrm{E}_{1}^{p-j, j}\right)=w_{p-j, j}$.

Conversely, assume that $f_{p j}$ are not all zero. Then "E degenerates at level two and we have

$$
\mathrm{h}^{p}\left(\mathcal{F} \otimes \Omega^{j}(j)\right)=\operatorname{dim}\left({ }^{\prime \prime} \mathrm{E}_{\infty}^{p}\right)=\operatorname{dim}\left({ }^{\prime \prime} \mathrm{E}_{2}^{p-j, j}\right)<\operatorname{dim}\left({ }^{\prime \prime} \mathrm{E}_{1}^{p-j, j}\right)=w_{p-j, j}
$$

for at least one choice of indices $p$ and $j$. This finishes the proof of the lemma.

Corollary 2. All the maps $\mathrm{H}^{i}\left(\mathcal{F} \otimes \Omega^{p}(p)\right) \otimes \mathcal{O}(-p) \longrightarrow$ $\longrightarrow \mathrm{H}^{i+1}\left(\mathcal{F} \otimes \Omega^{p}(p)\right) \otimes \mathcal{O}(-p)$ occuring in the Beilinson free monad for $\mathcal{F}$ are zero.

In the sequel we will assume that the schematic support of $\mathcal{F}$ has codimension $c$ in $\mathbb{P}^{n}$. The dual sheaf $\mathcal{F}^{\mathrm{D}}$ of $\mathcal{F}$ is defined by $\mathcal{F}^{\mathrm{D}}=\mathcal{E} x t^{c}\left(\mathcal{F}, \omega_{\mathrm{P}^{n}}\right)$. We remark that the hypothesis on the dimension of the support of $\mathcal{F}$ ensures that the extension sheaves $\mathcal{E} x t^{i}\left(\mathcal{F}, \omega_{\mathrm{p}^{n}}\right)$ vanish for $0 \leq i<c$, see (iii) 7.3 in [9].

Lemma 3. Let $\mathcal{F}$ be a coherent sheaf on $\mathrm{P}^{n}$ with support of codimension $c \geq 1$. Let

$$
0 \longrightarrow \mathcal{C}^{-p} \longrightarrow \ldots \longrightarrow \mathcal{C}^{0} \longrightarrow \ldots \longrightarrow \mathcal{C}^{q} \longrightarrow 0
$$

be a free monad for $\mathcal{F}$. Assume that $\mathcal{E} x t^{i}\left(\mathcal{F}, \omega_{\mathrm{p}^{n}}\right)=0$ for $i>c$. We consider the dual bundles $\mathcal{C}_{\mathrm{D}}^{i}=\mathcal{H}$ om $\left(\mathcal{C}^{-i-c}, \omega_{\mathrm{P}^{n}}\right)$. Then the dual sequence

$$
0 \longrightarrow \mathcal{C}_{\mathrm{D}}^{-q-c} \longrightarrow \ldots \longrightarrow \mathcal{C}_{\mathrm{D}}^{0} \longrightarrow \ldots \longrightarrow \mathcal{C}_{\mathrm{D}}^{p-c} \longrightarrow 0
$$

is a free monad for $\mathcal{F}^{\mathrm{D}}$.

Proof. We compare the spectral sequences 'E and "E converging to the hyper-derived functor $\mathcal{E} x t^{p+q}\left(\mathcal{C}^{*}, \omega_{\mathbb{P}^{n}}\right)$. They are given by

$$
\begin{aligned}
{ }^{\prime} \mathrm{E}_{1}^{p q} & =\mathcal{E} x t^{q}\left(\mathcal{C}^{-p}, \omega_{\mathbb{P}^{n}}\right), \\
{ }^{\prime \prime} \mathrm{E}_{2}^{p q} & =\mathcal{E} x t^{p}\left(\mathcal{H}^{-q}\left(\mathcal{C}^{*}\right), \omega_{\mathbb{P}^{n}}\right) .
\end{aligned}
$$

Since $\mathcal{C}^{i}$ are projective sheaves, we have ${ }^{\prime} \mathrm{E}_{1}^{p q}=0$ for $q \neq 0$. This shows that 'E degenerates at level two. The same is true of " $\mathrm{E}$ because ${ }^{\prime} \mathrm{E}_{2}^{p q}=0$ for $q \neq 0$. By virtue of the hypothesis and of the remark preceding the lemma, 
we have

$$
{ }^{\prime \prime} \mathrm{E}_{2}^{p 0}= \begin{cases}\mathcal{F}^{\mathrm{D}} & \text { for } p=c, \\ 0 & \text { for } p \neq c .\end{cases}
$$

In conclusion, the zero row of ' $\mathrm{E}_{1}$ provides the desired monad for $\mathcal{F}^{\mathrm{D}}$.

For any sheaf $\mathcal{F}$ on $\mathbb{P}^{n}$ there is a natural homomorphism $\mathcal{F} \longrightarrow \mathcal{F}^{\text {DD }}$ which is injective if and only if $\mathcal{F}$ is pure. We recall that a sheaf with support of dimension $d$ is called pure if it does not have a nonzero subsheaf with support of dimension smaller than $d$. We say that $\mathcal{F}$ is reflexive if the map $\mathcal{F} \longrightarrow \mathcal{F}^{\mathrm{DD}}$ is an isomorphism. According to 1.1 .10 in [12] the hypotheses of lemma 3 are satisfied for pure sheaves of dimension one and for reflexive sheaves of dimension two.

REMARK 4. Let $\mathcal{F}$ be a sheaf on $\mathbb{P}^{n}$ with support of dimension one. We assume that $\mathcal{F}$ is pure and we notice that this is equivalent to saying that $\mathcal{F}$ has no zero-dimensional torsion. According to lemma 3.1(i) and proposition 3.3(iv) from [7], this is further equivalent to saying that at every closed point $x$ in the support of $\mathcal{F}$ we have $\operatorname{depth}\left(\mathcal{F}_{x}\right) \geq 1$. From this we see that $\mathcal{F}$ satisfies Serre's condition $\mathrm{S}_{2, n-1}$ :

$$
\operatorname{depth}\left(\mathcal{F}_{x}\right) \geq \min \left\{2, \operatorname{dim}\left(\mathcal{O}_{\mathbb{P}^{n}, x}\right)-n+1\right\} \text { for all } x \in \operatorname{Supp}(\mathcal{F}) .
$$

From 1.1.10 in [12] we conclude that $\mathcal{F}$ is reflexive.

Proposition 5. Let $\mathcal{F}$ be a coherent sheaf on $\mathbb{P}^{n}$ with support of codimension $n-d=c \geq 1$. Assume that $\mathcal{E} x t^{i}\left(\mathcal{F}, \omega_{\mathbb{P}^{n}}\right)=0$ for $i>c$. Then for all $i, j$ we have

$$
\mathrm{h}^{i}\left(\mathcal{F} \otimes \Omega^{j}(j)\right)=\mathrm{h}^{d-i}\left(\mathcal{F}^{\mathrm{D}} \otimes \Omega^{n-j}(n-j+1)\right) .
$$

Proof. We apply lemma 3 to the Beilinson free monad for $\mathcal{F}$. We get a monad for $\mathcal{F}^{\mathrm{D}}$ with terms

$$
\mathcal{C}_{\mathrm{D}}^{i}=\mathcal{H o m}\left(\mathcal{C}^{-i-c}, \omega_{\mathbb{P}^{n}}\right)=\bigoplus_{0 \leq p \leq n} \mathrm{H}^{-i-c+p}\left(\mathcal{F} \otimes \Omega^{p}(p)\right)^{*} \otimes \mathcal{O}(p-n-1) .
$$

We tensor the above monad with $\mathcal{O}(1)$ to get a monad for $\mathcal{F}^{\mathrm{D}}(1)$. In view of corollary 2 , this monad satisfies the necessary and sufficient condition from lemma 1 . We conclude that for all $i, p$

$$
\mathrm{h}^{i+p}\left(\mathcal{F}^{\mathrm{D}} \otimes \Omega^{p}(p+1)\right)=\mathrm{h}^{d-i-p}\left(\mathcal{F} \otimes \Omega^{n-p}(n-p)\right) .
$$

This proves the proposition. 
Corollary 6. Let $\mathcal{F}$ be as in the previous proposition. Then for all $i, j$ we have

$$
\mathrm{h}^{i}(\mathcal{F})=\mathrm{h}^{d-i}\left(\mathcal{F}^{\mathrm{D}}\right)
$$

REMARK 7. The above corollary also follows from Serre duality and the degeneration of the local-to-global spectral sequence $\mathrm{E}_{2}^{p q}=\mathrm{H}^{p}\left(\mathbb{P}^{n}, \mathcal{E} x t^{q}\left(\mathcal{F}, \omega_{\mathbb{P}^{n}}\right)\right)$, which converges to $\operatorname{Ext}^{p+q}\left(\mathcal{F}, \omega_{\mathbb{P}^{n}}\right)$.

Next we would like to relate the Hilbert polynomials $P_{\mathcal{F}}$ and $P_{\mathcal{F}}$ of $\mathcal{F}$, respectively $\mathcal{F}^{\mathrm{D}}$. We recall that $P_{\mathcal{F}}$ has degree equal to the dimension of $\operatorname{Supp}(\mathcal{F})$. As the support of $\mathcal{F}^{\mathrm{D}}$ is included in the support of $\mathcal{F}$, we have $\operatorname{deg}\left(P_{\mathcal{F}^{\mathrm{D}}}\right) \leq \operatorname{deg}\left(P_{\mathcal{F}}\right)$.

Corollary 8. Let $\mathcal{F}$ be as in the previous proposition. Then for all $m$ we have

$$
P_{\mathcal{F}^{\mathrm{D}}}(m)=(-1)^{d} P_{\mathcal{F}}(-m) .
$$

In particular, if $\mathcal{F}$ is pure of dimension one with Hilbert polynomial $P_{\mathcal{F}}(m)=r m+\chi$, then the dual sheaf has Hilbert polynomial $P_{\mathcal{F}^{\mathrm{D}}}(m)=r m-\chi$.

LEMMA 9. Let $\mathcal{F}$ be a coherent sheaf on $\mathbb{P}^{n}, n \geq 2$, pure of dimension one. Then $\mathcal{F}$ is semistable (stable) if and only if $\mathcal{F}^{\mathrm{D}}$ is semistable (stable).

Proof. According to 1.2.6 in [12], the sheaf $\mathcal{F}$ is semistable (stable) if and only if for all pure one-dimensional quotients $\mathcal{G}$ of $\mathcal{F}$ we have

$$
\frac{\chi(\mathcal{G})}{r(\mathcal{G})} \geq(>) \frac{\chi(\mathcal{F})}{r(\mathcal{F})} .
$$

Take a pure one-dimensional destabilizing quotient $\mathcal{G}=\mathcal{F} / \mathcal{K}$. Since $\mathcal{K}$ has support of dimension one, we have $\mathcal{E} x t^{n-2}\left(\mathcal{K}, \omega_{\mathrm{p}}{ }^{n}\right)=0$, hence $\mathcal{G}^{\mathrm{D}}$ is a subsheaf of $\mathcal{F}^{\mathrm{D}}$. From corollary 8 we get

$$
\frac{\chi\left(\mathcal{G}^{\mathrm{D}}\right)}{r\left(\mathcal{G}^{\mathrm{D}}\right)}=-\frac{\chi(\mathcal{G})}{r(\mathcal{G})}>-\frac{\chi(\mathcal{F})}{r(\mathcal{F})}=\frac{\chi\left(\mathcal{F}^{\mathrm{D}}\right)}{r\left(\mathcal{F}^{\mathrm{D}}\right)} .
$$

Thus $\mathcal{G}^{\mathrm{D}}$ is a destabilizing subsheaf of $\mathcal{F}^{\mathrm{D}}$. This proves sufficiency. Necessity follows from the fact that $\mathcal{F}$ is reflexive, cf. remark 4 .

Two semistable sheaves $\mathcal{F}$ and $\mathcal{G}$ on $\mathrm{P}^{n}$ with Hilbert polynomial $P$ give the same point in $\mathrm{M}_{\mathbb{P}^{n}}(P)$ if and only if they are $S$-equivalent, meaning that 
there are filtrations by subsheaves

$$
\begin{gathered}
0=\mathcal{F}_{0} \subset \mathcal{F}_{1} \subset \ldots \subset \mathcal{F}_{\kappa-1} \subset \mathcal{F}_{\kappa}=\mathcal{F}, \\
0=\mathcal{G}_{0} \subset \mathcal{G}_{1} \subset \ldots \subset \mathcal{G}_{\kappa-1} \subset \mathcal{G}_{\kappa}=\mathcal{G},
\end{gathered}
$$

such that all quotients $\mathcal{F}_{i} / \mathcal{F}_{i-1}$ and $\mathcal{G}_{i} / \mathcal{G}_{i-1}$ are stable and there is a permutation $\sigma$ of the set of indices $\{1, \ldots, \kappa\}$ such that for all $i$

$$
\mathcal{F}_{i} / \mathcal{F}_{i-1} \simeq \mathcal{G}_{\sigma(i)} / \mathcal{G}_{\sigma(i)-1} .
$$

For stable sheaves S-equivalence means isomorphism. In other words, if the above conditions are satisfied, then $\mathcal{F}$ is stable if and only if $\mathcal{G}$ is stable and then $\kappa=1$ and $\mathcal{F} \simeq \mathcal{G}$. A Jordan-Hölder filtration of a semistable sheaf is a filtration as above by subsheaves such that all quotients are stable.

As in the proof of 9.3 from [14], a Jordan-Hölder filtration

$$
0=\mathcal{F}_{0} \subset \mathcal{F}_{1} \subset \ldots \subset \mathcal{F}_{\kappa-1} \subset \mathcal{F}_{\kappa}=\mathcal{F}
$$

for a semistable sheaf of dimension one on $\mathbb{P}^{n}$ gives a Jordan-Hölder filtration

$$
0=\left(\mathcal{F} / \mathcal{F}_{\kappa}\right)^{\mathrm{D}} \subset\left(\mathcal{F} / \mathcal{F}_{\kappa-1}\right)^{\mathrm{D}} \subset \ldots \subset\left(\mathcal{F} / \mathcal{F}_{1}\right)^{\mathrm{D}} \subset\left(\mathcal{F} / \mathcal{F}_{0}\right)^{\mathrm{D}}=\mathcal{F}^{\mathrm{D}}
$$

for the dual sheaf with quotients $\left(\mathcal{F}_{i} / \mathcal{F}_{i-1}\right)^{\mathrm{D}}$. The latter are stable by virtue of lemma 9 . We arrive at the following lemma:

LEmma 10. Let $\mathcal{F}$ and $\mathcal{G}$ be semistable sheaves on $\mathrm{P}^{n}$ with the same Hilbert polynomial $P(m)=r m+\chi$. If $\mathcal{F}$ and $\mathcal{G}$ are S-equivalent, then so are their duals.

LEMma 11. Let $S$ be an algebraic scheme over $k$ and let $\mathcal{F}$ be an $S$-flat coherent sheaf on $S \times \mathbb{P}^{n}$. Assume that for every $s$ in $S$ the restriction $\mathcal{F}_{s}=\mathcal{F}_{\mid\{s\} \times \mathrm{P}^{n}}$ has support of codimension $c$. Then for all $i<c$ we have

$$
\mathcal{E x t}^{i}\left(\mathcal{F}, \omega_{S \times \mathbb{P}^{n} / S}\right)=0 .
$$

Proof. Without loss of generality we may assume that $S$ is affine. As in the proof of (iii) 7.3 from [9], we reduce to showing the vanishing of $\operatorname{Ext}^{i}\left(\mathcal{F}, \omega_{S \times \mathrm{P}^{n} / S}(q)\right)$ for large $q$. By 11.2(f) on p. 213 of [8] we have the duality

$$
\operatorname{Ext}_{\mathcal{O}_{S \times \mathbb{p}}}^{i}\left(\mathcal{F}, \omega_{S \times \mathbb{P}^{n} / S}(q)\right) \simeq \operatorname{Hom}_{\mathcal{O}_{S}}\left(\mathrm{R}^{n-i} f_{*}(\mathcal{F}(-q)), \mathcal{O}_{S}\right)
$$

where $f: S \times \mathbb{P}^{n} \longrightarrow S$ is the projection onto the first component. As $n-i$ exceeds the dimension of each restriction $\mathcal{F}(-q)_{s}$, we have 
$\mathrm{H}^{n-i}\left(\mathcal{F}(-q)_{s}\right)=0$ for all $s$ in $S$. From exercise (iii) 11.8 in [9] we deduce that $\mathrm{R}^{n-i} f_{*}(\mathcal{F}(-q))=0$, so the above extension group vanishes.

In the sequel we will need the following relative version of the Hilbert syzygy theorem. Let $S$ be an algebraic scheme over $k$ and $\mathcal{F}$ a coherent $S$ flat sheaf on $S \times \mathbb{P}^{n}$. Then there exists a locally free resolution $\mathcal{E}^{\bullet} \longrightarrow \mathcal{F}$ of length at most $n$. Such a resolution can be constructed using the relative Beilinson spectral sequence with first term

$$
\mathrm{E}_{1}^{p q}=\mathrm{R}^{q} f_{*}\left(\mathcal{F}(m) \otimes g^{*} \Omega^{-p}(-p)\right) \bigotimes \mathcal{O}_{\mathrm{p}^{n}}(p),
$$

which converges to $\mathcal{F}(m)$ in degree zero and to 0 in degree different from zero (see 4.1.11 in [15]). Here $f: S \times \mathbb{P}^{n} \longrightarrow S$ and $g: S \times \mathbb{P}^{n} \longrightarrow \mathbb{P}^{n}$ are the canonical projections. If $m$ is large enough, then $\mathrm{E}_{1}^{p q}=0$ for $q \neq 0$ and the push-forward sheaves $f_{*}\left(\mathcal{F}(m) \otimes g^{*} \Omega^{-p}(-p)\right)$ are locally free. Thus the spectral sequence degenerates at level two and the zero row of $\mathrm{E}_{1}$ provides a locally free resolution of $\mathcal{F}(m)$. We can cover $S$ with open affine subsets $U$, such that the restriction of each term of the resolution to $U \times \mathbb{P}^{n}$ is a direct sum of line bundles of the form $g^{*} \mathcal{O}_{\mathrm{p}^{n}}(-l)$.

LeMma 12. Let $S$ be an algebraic scheme over $k$ and let $\mathcal{F}$ be an $S$-flat coherent sheaf on $S \times \mathbb{P}^{n}$. Assume that for every $s$ in $S$ the restriction $\mathcal{F}_{s}=\mathcal{F}_{\mid\{s\} \times \mathbb{P}^{n}}$ has support of codimension $c$ and that $\mathcal{E} \mathrm{t}^{i}\left(\mathcal{F}_{s}, \omega_{\mathbb{P}^{n}}\right)=0$ for $i>c$. Then

$$
\mathcal{E} x t^{i}\left(\mathcal{F}, \omega_{S \times \mathbb{P}^{n} / S}\right)=0 \text { for } \quad i \neq c .
$$

Moreover, the sheaf $\mathcal{F}^{\mathrm{D}}=\mathcal{E} x t^{c}\left(\mathcal{F}, \omega_{S \times \mathbb{P}^{n} / S}\right)$ is flat over $S$ and $\left(\mathcal{F}_{s}\right)^{\mathrm{D}} \simeq\left(\mathcal{F}^{\mathrm{D}}\right)_{s}$ for all s in $S$.

Proof. Consider a finite locally free resolution $\mathcal{E}^{\bullet} \longrightarrow \mathcal{F}$. The extension sheaf $\mathcal{E x t}^{p}\left(\mathcal{F}, \omega_{S \times \mathrm{P}^{n} / S}\right)$ is the $p$-th cohomology of the complex $\mathcal{H o m}\left(\mathcal{E}^{*}, \omega_{S \times \mathbb{P}^{n} / S}\right)$. We fix a point $s$ in $S$ and denote $i:\{s\} \times \mathbb{P}^{n} \longrightarrow S \times \mathbb{P}^{n}$ the canonical inclusion. The functor $\mathcal{H o m}\left({ }_{-}, \omega_{S \times \mathbb{P}^{n} / S}\right)$ sends projective sheaves to $i^{*}$-acyclic sheaves, hence there is the Grothendieck spectral sequence (see (viii) 9.3 in [10])

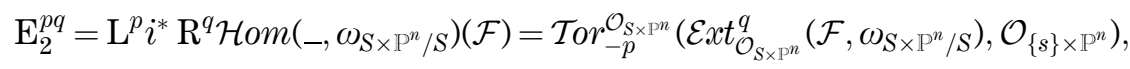

which converges to the hyper-derived functors $\mathbb{L}^{p+q} i^{*}\left(\mathcal{H o m}\left(\mathcal{E}^{\bullet}, \omega_{S \times \mathbb{P}^{n} / S}\right)\right)$. We recall that the above is the second term of the spectral sequence associated to the bigraded complex obtained by taking a fourth-quadrant Cartan-Eilenberg resolution of $\mathcal{H o m}\left(\mathcal{E}^{\bullet}, \omega_{S \times \mathbb{P}^{n} / S}\right)$ and then applying $i^{*}$. The 
other spectral sequence associated to the bigraded complex is given by

$$
{ }^{\prime} \mathrm{E}_{1}^{p q}=\mathrm{L}^{q} i^{*}\left(\mathcal{H o m}\left(\mathcal{E}^{-p}, \omega_{S \times \mathbb{P}^{n} / S}\right)\right) .
$$

'E degenerates at level two because ${ }^{\prime} \mathrm{E}_{1}^{p q}=0$ for $q \neq 0$. Thus

$$
\mathbb{L}^{p} i^{*}\left(\mathcal{H o m}\left(\mathcal{E}^{\cdot}, \omega_{S \times \mathbb{P}^{n} / S}\right)\right) \simeq{ }^{\prime} \mathrm{E}_{\infty}^{p 0}={ }^{\prime} \mathrm{E}_{2}^{p 0}
$$

is the cohomology at position $i^{*} \mathcal{H} \operatorname{Hom}\left(\mathcal{E}^{-p}, \omega_{S \times \mathbb{P}^{n} / S}\right)$ of the complex

$$
i^{*} \mathcal{H o m}\left(\mathcal{E}^{\bullet}, \omega_{S \times \mathbb{P}^{n} / S}\right) \simeq \mathcal{H o m}\left(i^{*} \mathcal{E}^{\bullet}, i^{*} \omega_{S \times \mathbb{P}^{n} / S}\right) \simeq \mathcal{H o m}\left(\mathcal{E}_{s}, \omega_{\mathbb{P}^{n}}\right) .
$$

As every term of the resolution $\mathcal{E}^{\bullet} \longrightarrow \mathcal{F}$ is $S$-flat, restricting to $\{s\} \times \mathbb{P}^{n}$ we obtain a locally free resolution $\mathcal{E}_{s}^{\bullet} \longrightarrow \mathcal{F}_{s}$. Thus, for all $j$,

$$
\mathbb{L}_{i}^{j} i^{*}\left(\mathcal{H o m}\left(\mathcal{E}^{\bullet}, \omega_{S \times \mathbb{P}^{n} / S}\right)\right) \simeq \mathcal{E} x t^{j}\left(\mathcal{F}_{s}, \omega_{\mathbb{P}^{n}}\right) .
$$

By hypothesis the above sheaves are zero for $j \neq c$, forcing $\mathrm{E}_{\infty}^{p q}=0$ for $p+q \neq c$. Assume $c<n$. From the vanishing of

$$
\mathrm{E}_{\infty}^{0 n}=\mathrm{E}_{2}^{0 n}=\mathcal{E} x t^{n}\left(\mathcal{F}, \omega_{S \times \mathbb{P}^{n} / S}\right)_{s}
$$

for an arbitrary point $s$ in $S$, we deduce that $\mathcal{E} x t^{n}\left(\mathcal{F}, \omega_{S \times \mathbb{P}^{n} / S}\right)=0$. Thus $\mathrm{E}_{2}^{p n}=0$ for all $p$, forcing $\mathrm{E}_{2}^{0, n-1}=\mathrm{E}_{\infty}^{0, n-1}$. If $c<n-1$ we can repeat the argument and conclude that $\mathcal{E} x t^{n-1}\left(\mathcal{F}, \omega_{S \times \mathbb{P}^{n} / S}\right)=0$. By induction we obtain that the sheaves $\mathcal{E} x t^{q}\left(\mathcal{F}, \omega_{S \times \mathbb{P}^{n} / S}\right)$ vanish for $q>c$. In view of lemma 11 they also vanish for $q<c$. Thus $\mathrm{E}_{2}^{p q}=0$ for $q \neq c$ and $\mathrm{E}$ degenerates at level two:

$$
\mathrm{E}_{2}^{p c}=\mathrm{E}_{\infty}^{p c} \simeq \mathbb{L}^{p+c} i^{*}\left(\mathcal{H} \text { om }\left(\mathcal{E}^{\bullet}, \omega_{S \times \mathbb{P}^{n} / S}\right)\right) \simeq \mathcal{E} x t^{p+c}\left(\mathcal{F}_{s}, \omega_{\mathbb{P}^{n}}\right) .
$$

For $p=0$ we arrive at the isomorphism $\left(\mathcal{F}^{\mathrm{D}}\right)_{s} \simeq\left(\mathcal{F}_{s}\right)^{\mathrm{D}}$. For $p=-1$ we get

$$
\mathcal{T o r}_{1}^{\mathcal{O}_{S \times \mathbb{P}^{n}}}\left(\mathcal{F}^{\mathrm{D}}, \mathcal{O}_{\{s\} \times \mathbb{P}^{n}}\right)=0 .
$$

From this we deduce that $\mathcal{F}^{\mathrm{D}}$ is $S$-flat. Indeed, localizing at a point $x$ lying over $s$, we obtain

$$
\operatorname{Tor}_{1}^{\mathcal{O}_{x}}\left(\left(\mathcal{F}^{\mathrm{D}}\right)_{x}, \mathcal{O}_{x} \otimes_{\mathcal{O}_{s}} k(s)\right)=0 .
$$

Here $k(s)$ denotes the residue field of $s$. We now use the local criterion of flatness 4.1.3 in [13] to conclude that the stalk $\left(\mathcal{F}^{\mathrm{D}}\right)_{x}$ is flat over $\mathcal{O}_{s}$.

Before we proceed any further we need to review the construction of the moduli space $\mathrm{M}_{\mathbb{P}^{n}}(P)$ of semistable sheaves on $\mathbb{P}^{n}$ with fixed Hilbert polynomial $P$. There exists an integer $m \gg 0$ such that for every semistable sheaf $\mathcal{F}$ on $\mathbb{P}^{n}$ with Hilbert polynomial $P$ the twisted sheaf $\mathcal{F}(m)$ is generated by global sections and the higher cohomology groups $\mathrm{H}^{i}(\mathcal{F}(m))$, $i \geq 1$, vanish. Thus $\mathrm{h}^{0}(\mathcal{F}(m))=P(m)$ and $\mathcal{F}$ occurs as a quotient 
$\rho: V \otimes \mathcal{O}(-m) \rightarrow \mathcal{F}$, where $V$ is a fixed vector space over $k$ of dimension $P(m)$. We consider the quotient scheme $Q=\operatorname{Quot}_{\mathrm{p}^{n}}\left(V \otimes \mathcal{O}_{\mathbb{P}^{n}}(-m), P\right)$ and the universal quotient sheaf $\widetilde{\mathcal{F}}$ on $Q \times \mathbb{P}^{n}$. Let $R \subset Q$ be the open subset of equivalence classes of quotients $\left[\rho: V \otimes \mathcal{O}_{\mathbb{P}^{n}}(-m) \rightarrow \mathcal{F}\right]$ for which $\mathcal{F}$ is semistable and the map on global sections $\mathrm{H}^{0}(\rho(m)): V \longrightarrow \mathrm{H}^{0}(\mathcal{F}(m))$ is an isomorphism. The reductive group $\mathrm{SL}(V)$ acts on $Q$ via its action on the first component of $V \otimes \mathcal{O}_{\mathrm{P}^{n}}(-m)$. Clearly $R$ is invariant under this action. If $m$ is chosen large enough, then $\mathrm{M}_{\mathbb{P}^{n}}(P)$ is a good quotient of $R$ by $\mathrm{SL}(V)$. We denote by $\pi: R \longrightarrow \mathrm{M}_{\mathbb{P}^{n}}(P)$ be the good quotient map.

THEOREM 13. For any integers $n \geq 2, r \geq 1$ and $\chi$ there is an isomorphism

$$
\mathrm{M}_{\mathbb{P}^{n}}(r, \chi) \longrightarrow \mathrm{M}_{\mathbb{P}^{n}}(r,-\chi)
$$

which maps the S-equivalence class of a sheaf $\mathcal{F}$ to the S-equivalence class of $\mathcal{F}^{\mathrm{D}}$.

Proof. According to remark 4, corollary 8, lemma 9 and lemma 10 the above map, call it $\eta$, is well-defined and bijective. It remains to show that $\eta$ is a morphism. By symmetry it will follow that $\eta^{-1}$ is also a morphism.

We put $P(m)=r m+\chi$, so $P^{\mathrm{D}}(m)=r m-\chi$. We adopt the notations preceeding the theorem. The sheaf $\widetilde{\mathcal{F}}^{\mathrm{D}}$ on $R \times \mathbb{P}^{n}$ defined by $\widetilde{\mathcal{F}}^{\mathrm{D}}=$ $=\mathcal{E} x t^{n-1}\left(\widetilde{\mathcal{F}}, \omega_{R \times \mathbb{P}^{n} / R}\right)$ is coherent and, by the previous lemma, $R$-flat. Moreover, for each $s$ in $R$, its restriction $\widetilde{\mathcal{F}}_{s}^{\mathrm{D}}$ to $\{s\} \times \mathrm{P}^{n}$ is isomorphic to $\left(\widetilde{\mathcal{F}}_{s}\right)^{\mathrm{D}}$. According to corollary 8 , the latter has Hilbert polynomial $P^{\mathrm{D}}$. The moduli space property of $\mathrm{M}_{\mathrm{P}^{n}}\left(P^{\mathrm{D}}\right)$ gives a morphism $\pi^{\mathrm{D}}: R \longrightarrow \mathrm{M}_{\mathrm{P}^{n}}\left(P^{\mathrm{D}}\right)$ which maps $s$ to the S-equivalence class of $\widetilde{\mathcal{F}}_{s}^{\mathrm{D}}$.

From lemma 10 we see that $\pi^{\mathrm{D}}$ is constant on the fibers of $\pi$. The good quotient property of $\pi$ shows that $\pi^{\mathrm{D}}$ factors through a morphism $\mathrm{M}_{\mathbb{P}^{n}}(P) \longrightarrow \mathrm{M}_{\mathbb{P}^{n}}\left(P^{\mathrm{D}}\right)$. This morphism is $\eta$, which finishes the proof of the theorem.

REMARK 14. If $P$ has degree at least 2 , then the above proof yields a morphism

$$
U \longrightarrow \mathrm{M}_{\mathrm{P}^{n}}\left(P^{\mathrm{D}}\right), \quad[\mathcal{F}] \longrightarrow\left[\mathcal{F}^{\mathrm{D}}\right]
$$

defined on the open set $U$ in $\mathrm{M}_{\mathrm{P}^{n}}(P)$ of isomorphism classes of stable sheaves $\mathcal{F}$ with $\mathcal{F}^{\mathrm{D}}$ stable and satisfying $\mathcal{E} x t^{i}\left(\mathcal{F}, \omega_{\mathrm{P}^{n}}\right)=0$ for $i>c$. For sheaves supported on surfaces the last condition is equivalent to saying that $\mathcal{F}$ is reflexive. We notice that $U$ is nonempty for all $P$ of degree 2 for which 
there is a smooth surface $X$ in $\mathbb{P}^{n}$ and a line bundle $\mathcal{L}$ on $X$ with Hilbert polynomial $P$. This is so because any locally free $\mathcal{O}_{X}$-module of rank one, with $X$ a reduced subscheme of $\mathbb{P}^{n}$, is stable as a sheaf on $\mathbb{P}^{n}$. Moreover, $\mathcal{L}$ is reflexive as a sheaf on $\mathbb{P}^{n}$, which can be seen from the isomorphism $\left(i_{*} \mathcal{L}\right)^{\mathrm{D}} \simeq i_{*}\left(\mathcal{L}^{\mathrm{D}}\right)$, where $i: X \longrightarrow \mathbb{P}^{n}$ is the embedding map.

In the remaining part of this paper we will apply the duality result to the following situation. Assume that $\mathrm{M}_{\mathrm{P}^{n}}(r, \chi)$ is the quotient of a certain parameter space by an algebraic group. This could be the case, for instance, if all sheaves giving a point in $\mathrm{M}_{\mathrm{p}^{n}}(r, \chi)$ are quasi-isomorphic to monads of a certain kind. Constructions of moduli spaces of sheaves as quotients of parameter spaces are ubiquitous in the literature, mostly involving actions of reductive groups, and, more recently, as in [2], [3] or [6], for actions of nonreductive groups. Under the above hypothesis on $\mathrm{M}_{\mathrm{p}^{n}}(r, \chi)$, we will show that $\mathrm{M}_{\mathrm{p}^{n}}(r,-\chi)$ is the quotient of the "dual parameter space" modulo the "dual group." This will generalize our partial results from section 9 of [14], where we dealt with locally closed subvarieties inside $\mathrm{M}_{\mathrm{p}^{2}}(r, \chi)$ for certain choices of $r$ and $\chi$.

We fix sheaves $\mathcal{C}^{i}$ on $\mathbb{P}^{n},-p \leq i \leq q$, that are finite direct sums of line bundles. We fix a polynomial $P(m)=r m+\chi$. We assume that each semistable sheaf on $\mathbb{P}^{n}$ with Hilbert polynomial $P(m)=r m+\chi$ occurs as the cohomology of a monad

$$
0 \longrightarrow \mathcal{C}^{-p} \stackrel{\varphi_{-p}}{\longrightarrow} \ldots \stackrel{\varphi_{-1}}{\longrightarrow} \mathcal{C}^{0} \stackrel{\varphi_{0}}{\longrightarrow} \ldots \stackrel{\varphi_{q-1}}{\longrightarrow} \mathcal{C}^{q} \longrightarrow 0 .
$$

For a monad of this form we write $\varphi=\left(\varphi_{-p}, \ldots, \varphi_{q-1}\right)$ and we denote by $\mathcal{F}_{\varphi}$ its cohomology. Let $W^{s s}$ be the set of all those $\varphi$ for which $\mathcal{F}_{\varphi}$ is semistable. We assume that $W^{s s}$ is a constructible subset inside the finite dimensional vector space

$$
W=\operatorname{Hom}\left(\mathcal{C}^{-p}, \mathcal{C}^{-p+1}\right) \times \ldots \times \operatorname{Hom}\left(\mathcal{C}^{q-1}, \mathcal{C}^{q}\right) .
$$

We equip $W^{s s}$ with the induced structure of a reduced variety. The algebraic group

$$
G=\operatorname{Aut}\left(\mathcal{C}^{-p}\right) \times \ldots \times \operatorname{Aut}\left(\mathcal{C}^{q}\right)
$$

acts on $W$ in an obvious manner: given $g=\left(g_{-p}, \ldots, g_{q}\right)$ and a tuple $\varphi$ as above we put

$$
g \varphi=\left(g_{-p+1} \varphi_{-p} g_{-p}^{-1}, \ldots, g_{q} \varphi_{q-1} g_{q-1}^{-1}\right) .
$$

We remark that, if at least one $\mathcal{C}^{i}$ has a direct summand of the form $\mathcal{O}_{\mathbb{P}^{n}}(a) \oplus \mathcal{O}_{\mathbb{P}^{n}}(b), a<b$, then $G$ is nonreductive, i.e. it has a nontrivial uni- 
potent radical, cf. 19.5 in [11]. Indeed, the subgroup of $\operatorname{Aut}\left(\mathcal{O}_{\mathbb{P}^{n}}(a) \oplus \mathcal{O}_{\mathbb{P}^{n}}(b)\right)$ given by matrices of the form

$$
\left[\begin{array}{ll}
1 & 0 \\
\star & 1
\end{array}\right]
$$

is a normal connected unipotent subgroup. This shows that the unipotent radical of $\operatorname{Aut}\left(\mathcal{O}_{\mathbb{P}^{n}}(a) \oplus \mathcal{O}_{\mathbb{P}^{n}}(b)\right)$ is nontrivial. A similar argument applies in general to $G$.

Clearly $\mathcal{F}_{\varphi}$ and $\mathcal{F}_{g \varphi}$ are isomorphic, so one is semistable if and only if the other is. This shows that $W^{s s}$ is $G$-invariant.

Let $p: W^{s s} \times \mathbb{P}^{n} \longrightarrow \mathbb{P}^{n}$ be the projection onto the second component. We put $\widetilde{\mathcal{C}}^{i}=p^{*} \mathcal{C}^{i}$. On $W^{s s} \times \mathbb{P}^{n}$ we have the universal monad

$$
\widetilde{\mathcal{C}}^{-p} \stackrel{\Phi_{-p}}{\longrightarrow} \ldots \stackrel{\Phi_{-1}}{\longrightarrow} \widetilde{\mathcal{C}}^{0} \stackrel{\Phi_{0}}{\longrightarrow} \ldots \stackrel{\Phi_{q-1}}{\longrightarrow} \widetilde{\mathcal{C}}^{q}
$$

with $\Phi_{i \mid\{\varphi\} \times \mathbb{P}^{n}}=\varphi_{i}$. Let $\widetilde{\mathcal{F}}$ denote the middle cohomology of this sequence. Using arguments as in the proof of lemma 12 one can see that its restriction $\widetilde{\mathcal{F}}_{\varphi}$ to any fiber $\{\varphi\} \times \mathbb{P}^{n}$ is isomorphic to $\mathcal{F}_{\varphi}$. In particular, all $\widetilde{\mathcal{F}}_{\varphi}$ have the same Hilbert polynomial $P$. As $W^{s s}$ is reduced we deduce that $\widetilde{\mathcal{F}}$ is $W^{s s}$-flat. The moduli space property of $\mathrm{M}_{\mathrm{P}^{n}}(P)$ yields a morphism

$$
\pi: W^{s s} \longrightarrow \mathrm{M}_{\mathbb{P}^{n}}(P), \quad \pi(\varphi)=\left[\mathcal{F}_{\varphi}\right]
$$

Clearly $\pi$ is $G$-equivariant. We will be interested in the case in which $\pi$ is a good or a geometric quotient.

We now apply the duality result to the above set-up. Under the hypothesis that each sheaf giving a point in $\mathrm{M}_{\mathbb{P}^{n}}(P)$ is the cohomology of a monad $\mathcal{C}^{*}$ and by virtue of lemma 3 , corollary 8 and lemma 9 , we see that each semistable sheaf on $\mathbb{P}^{n}$ with Hilbert polynomial $P^{\mathrm{D}}(m)=r m-\chi$ is the cohomology of a monad

$$
0 \longrightarrow \mathcal{C}_{\mathrm{D}}^{-q-c} \stackrel{\varphi_{-q-c}^{\mathrm{D}}}{\longrightarrow} \ldots \stackrel{\varphi_{-1}^{\mathrm{D}}}{\longrightarrow} \mathcal{C}_{\mathrm{D}}^{0} \stackrel{\varphi_{0}^{\mathrm{D}}}{\longrightarrow} \ldots \stackrel{\varphi_{p-c-1}^{\mathrm{D}}}{\longrightarrow} \mathcal{C}_{\mathrm{D}}^{p-c} \longrightarrow 0
$$

with

$$
\mathcal{C}_{\mathrm{D}}^{i}=\mathcal{H o m}\left(\mathcal{C}^{-i-c}, \omega_{\mathbb{P}^{n}}\right), \quad \varphi_{i}^{\mathrm{D}}=\mathcal{H} \operatorname{Hom}\left(\varphi_{-i-c-1}, \omega_{\mathrm{P}^{n}}\right)
$$

We let $W_{\mathrm{D}}^{s s}$ be the set of all those tuples $\varphi^{\mathrm{D}}=\left(\varphi_{-q-c}^{\mathrm{D}}, \ldots, \varphi_{p-c-1}^{\mathrm{D}}\right)$ for which $\mathcal{F}_{\varphi^{\mathrm{D}}}$ is semistable. We view $W_{\mathrm{D}}^{s s}$ as a subvariety inside the affine variety

$$
W_{\mathrm{D}}=\operatorname{Hom}\left(\mathcal{C}_{\mathrm{D}}^{-q-c}, \mathcal{C}_{\mathrm{D}}^{-q-c+1}\right) \times \ldots \times \operatorname{Hom}\left(\mathcal{C}_{\mathrm{D}}^{p-c-1}, \mathcal{C}_{\mathrm{D}}^{p-c}\right)
$$


equipped with the analogously defined action of the group of automorphisms

$$
G_{\mathrm{D}}=\operatorname{Aut}\left(\mathcal{C}_{\mathrm{D}}^{-q-c}\right) \times \ldots \times \operatorname{Aut}\left(\mathcal{C}_{\mathrm{D}}^{p-c}\right) .
$$

As before, there is a $G_{\mathrm{D}}$-equivariant map

$$
\pi^{\mathrm{D}}: W_{\mathrm{D}}^{s s} \longrightarrow \mathrm{M}_{\mathrm{P}^{n}}\left(P^{\mathrm{D}}\right), \quad \pi^{\mathrm{D}}\left(\varphi^{\mathrm{D}}\right)=\left[\mathcal{F}_{\varphi^{\mathrm{D}}}\right] .
$$

COROLLARY 15. $\pi$ is a good (geometric) quotient map if and only if $\pi^{\mathrm{D}}$ is a good (geometric) quotient map.

Proof. First we notice that $G \simeq G_{\mathrm{D}}$ and that the map $\varphi \longrightarrow \varphi^{\mathrm{D}}$ acts by transposition, so it gives an equivariant isomorphism $W^{s s} \longrightarrow W_{\mathrm{D}}^{s s}$. According to lemma 3 this isomorphism fits into a commutative diagram

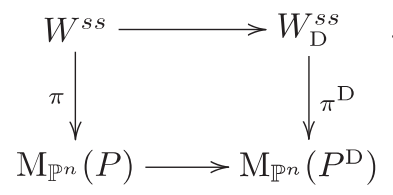

By virtue of the previous theorem the bottom map $[\mathcal{F}] \longrightarrow\left[\mathcal{F}^{\mathrm{D}}\right]$ is an isomorphism, which proves the statement.

We finish with an example. Fix a vector space $V$ over $k$ of dimension 4 . According to [6] a sheaf $\mathcal{F}$ on $\mathbb{P}^{3}=\mathbb{P}(V)$ with Hilbert polynomial $3 m+1$ is semistable if and only if it has a resolution

$$
0 \longrightarrow 2 \mathcal{O}(-3) \stackrel{\psi}{\longrightarrow} \mathcal{O}(-1) \oplus 3 \mathcal{O}(-2) \stackrel{\varphi}{\longrightarrow} \mathcal{O} \oplus \mathcal{O}(-1) \longrightarrow \mathcal{F} \longrightarrow 0
$$

with $\varphi$ not equivalent to a matrix of the form

$$
\left[\begin{array}{llll}
\star & \star & \star & \star \\
0 & 0 & \star & \star
\end{array}\right]
$$

Moreover, $\mathrm{M}_{\mathrm{p}^{3}}(3,1)$ is a geometric quotient of the space of parameters $(\psi, \varphi)$ modulo the action of the group of automorphisms. The concrete description of the space of parameters $W^{s s}$ is given at 5.2 in [6]. It consists of pairs $(\psi, \varphi)$ for which the sequence of global sections

$$
0 \longrightarrow k^{2} \stackrel{\mathrm{H}^{0}(\psi(3))}{\longrightarrow} S^{2} V^{*} \oplus\left(k^{3} \otimes V^{*}\right) \stackrel{\mathrm{H}^{0}(\varphi(3))}{\longrightarrow} S^{3} V^{*} \oplus S^{2} V^{*}
$$

is exact and either $\varphi_{21} \neq 0$ or the entries $\left\{\varphi_{22}, \varphi_{23}, \varphi_{24}\right\}$ are linearly independent as elements of $V^{*}$. 
The above considerations tell us that each sheaf $\mathcal{F}$ on $\mathrm{P}^{3}$ with Hilbert polynomial $3 m-1$ is semistable if and only if there is $(\psi, \varphi)$ in $W^{s s}$ and a resolution

$$
0 \longrightarrow \mathcal{O}(-4) \oplus \mathcal{O}(-3) \stackrel{\varphi^{\mathrm{D}}}{\longrightarrow} \mathcal{O}(-3) \oplus 3 \mathcal{O}(-2) \stackrel{\psi^{\mathrm{D}}}{\longrightarrow} 2 \mathcal{O}(-1) \longrightarrow \mathcal{F} \longrightarrow 0 .
$$

From the above corollary we deduce that $\mathrm{M}_{\mathrm{P}^{3}}(3,-1)$ is a geometric quotient of the space of parameters $W_{\mathrm{D}}^{s s}$ of transposed matrices $\left(\varphi^{\mathrm{D}}, \psi^{\mathrm{D}}\right)$ modulo the group of automorphisms.

Acknowledgements. The author thanks the referee for providing several corrections and improvements and for numerous helpful comments. The referee suggested the proof of lemma 12, shortened our original proof of lemma 3 and pointed out that the two conditions from lemma 1 are equivalent (originally we stated only sufficiency).

\section{REFERENCES}

[1] Aleksandr Beilinson, Coherent Sheaves on $\mathrm{P}^{n}$ and Problems of Linear Algebra, Func. Anal. Appl., 12 (1979), pp. 214-216. (Translation of Funktsional. Anal. i Prilozhen. 12 (1978), pp. 68-69.)

[2] Jean-Marc Drézet, Variétés de modules extrémales de faisceaux semistables sur $\mathbb{P}_{2}(\mathrm{C})$, Math. Ann., 290 (1991), pp. 727-770.

[3] Jean-Marc Drézet - Günther Trautmann, Moduli Spaces of Decomposable Morphisms of Sheaves and Quotients by Non-Reductive Groups, Ann. Inst. Fourier, 53 (2003), pp. 107-192.

[4] David Eisenbud - Gunnar Fløystad - Frank-Olaf Schreyer, Sheaf Cohomology and Free Resolutions over Exterior Algebras, Trans. Am. Math. Soc., 355 (2003), pp. 4397-4426.

[5] Hans-Georg Freiermuth, On the Moduli Space $M_{P}\left(\mathbb{P}_{3}\right)$ of Semi-stable Sheaves on $\mathbb{P}_{3}$ with Hilbert Polynomial $P(m)=3 m+1$, Diplomarbeit at Univ. Kaiserslautern (December, 2000).

[6] Hans-Georg Freiermuth - GÜnther Trautmann, On the Moduli Scheme of Stable Sheaves Supported on Cubic Space Curves, Amer. J. Math., 126 (2004), pp. 363-393.

[7] Alexandre Grothendieck, Cohomologie locale des faisceaux cohérents et théorèmes de Lefschetz locaux et globaux, Séminaire de Géométrie Algébrique du Bois Marie 1962, Societé Mathématique de France, e-print 0511279.

[8] Robin Hartshorne, Residues and Duality, Lecture Notes in Mathematics, 20 (Springer Verlag, Berlin, 1966).

[9] Robin Hartshorne, Algebraic Geometry, Graduate Texts in Mathematics, 52 (Springer Verlag, New York, 1977).

[10] Peter Hilton - Urs Stammbach, A Course in Homological Algebra, Graduate Texts in Mathematics, 4 (Springer Verlag, New York, 1971). 
[11] James Humphreys, Linear Algebraic Groups, Graduate Texts in Mathematics, 21 (Springer Verlag, New York, 1975).

[12] Daniel Huybrechts - Manfred Lehn, The Geometry of Moduli Spaces of Sheaves, Aspects of Mathematics, E31 (Friedr. Vieweg Sohn, Braunschweig, 1997).

[13] Joseph Le Potier, Lectures on Vector Bundles, Cambridge University Press, 1997, translated by A. Maciocia.

[14] Mario Maican, On Two Notions of Semistability, Pacific J. Math., 234 (2008), pp. $69-135$.

[15] Christian Okonek - Michael Schneider - Heinz Spindler, Vector Bundles on Complex Projective Spaces, Progress in Mathematics, 3 (Birkhäuser, Boston, 1980).

Manoscritto pervenuto in redazione il 23 gennaio 2009. 